\title{
FluidDissipation for Applications - A Library for Modelling of Heat Transfer and Pressure Loss in Energy Systems
}

\author{
Thorben Vahlenkamp Stefan Wischhusen \\ XRG Simulation $\mathrm{GmbH}$, \\ Harburger Schlossstraße 6-12, 21079 Hamburg \\ \{vahlenkamp, wischhusen\}@xrg-simulation.de
}

\begin{abstract}
The results of a free and open source ModELICA library for convective heat transfer and pressure loss calculations of energy devices called FLUIDDISSIPATION will be presented based on the goals shown at the Modelica Conference 2008 [1]. The FluidDissiPATION library is developed within the European research project EuroSysLib.
\end{abstract}

The library delivers a broad range of verified and validated correlations describing convective heat transfer and pressure loss of fluids in energy devices. These correlations are numerically optimised to provide efficient and stable transient simulations. The library also provides convective heat transfer models and flow models of most heat transfer and pressure loss correlations using the also free and open source MODELICA_FLUID library [2] as thermo-hydraulic framework for system simulation.

Scope, implementation concept, numerical challenges, verification and validation of the FLUIDDISSIPATION library will be exemplarily described (e.g. for convective heat transfer and pressure loss of twophase flow).

Industrial applications for thermo-hydraulic system simulation (e.g. air distribution circuit for supplemental cooling, aircraft engine fuel feeding system) are presented using FLUIDDISSIPATION correlations implemented within ModELICA_FLUID models. A detailed documentation is available in the library itself.

Keywords: convective heat transfer; pressure loss; dissipation; ModELICA_FLUID

\section{Introduction}

Energy conversion in any thermo-hydraulic process is declined due to unwanted heat transfer (as a result of temperature difference) and pressure losses (as a result of local losses due to geometry and/or frictional losses) of a working fluid [3]. Both physical phenomena increase entropy and decrease exergy of an energy system. Therefore an amount of energy of a working fluid to be transformed into mechanical work is dissipated.

These fluid dissipation effects (e.g. pressure loss of pipe network) have to be compensated by higher energy supply of other system components (e.g. delivery height of pumps). A reduction of fluid dissipation effects is a way to optimise the efficiency of a thermohydraulic process with a corresponding minimisation of operation costs.

Thus modelling fluid dissipation effects are necessary for thermo-hydraulic processes to evaluate existing energy systems and to find out optimising potentials.

The following sections deliver insight into the open source MODELICA library FLUIDDISSIPATION:

- Library content with verified and validated correlations describing the dissipation effects due to convective heat transfer and pressure losses of fluids used in energy devices

- Implementation of the library describing its functional approach, numerical optimisations and the creation of convective heat transfer and flow models (out of MODELICA_FLUID as thermohydraulic framework and the dissipation correlations)

- Verification and validation of the library

- Convective heat transfer is exemplarily validated for an even gap as well as for condensation and boiling of two-phase flow 
- Pressure loss is exemplarily validated for a curved bend as well as for two-phase flow

- System simulation of an environmental control system, a supplemental cooling system and an aircraft engine feeding system as industrial application

\section{Library content}

The FLuidDissipation library consists of five major packages shown in Fig.1.

(1) UluidDissipation
$\square$ Examples
bHeatTransfer
bPressureLoss
$\square$ Utilities

Figure 1: Package content of the FLUIDDISSIPATION library.

The USERS GUIDE section provides a GETTINGSTARTED for the usage of the FluidDissipation library and reports important changes w.r.t. previous versions.

The EXAMPLES package is divided into three packages. In the VERIFICATIONS package all heat transfer and pressure loss correlations are verified or validated with literature. In the APPLICATION package these correlations are implemented into MODELICA_FLUID as thermo-hydraulic framework. Examples of convective heat transfer models and flow models are provided. In the TESTCASES package these MODELICA_FLUID models are used for system simulation of test applications (e.g. aircraft engine feeding system).

The HeatTransfer section consists of 6 major energy device packages shown in Fig.2. Each energy device package contains its corresponding correlations for the convective heat transfer coefficient. Every package can provide several correlations for this device (best seen in the library itself). For example a channel can be calculated with a constant wall temperature or constant heat flow rate as boundary condition.

The Pressureloss section consists of 9 major energy device packages shown in Fig.3. Each energy device package contains its corresponding correlations for the pressure loss coefficient. Every package can provide several correlations for this device (best seen

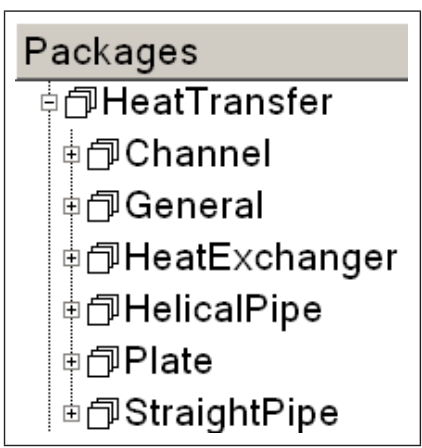

Figure 2: Package content of convective heat transfer devices in the FLUIDDISSIPATION library.

in the library itself). For example a bend can be calculated with a curved or an edged turning.

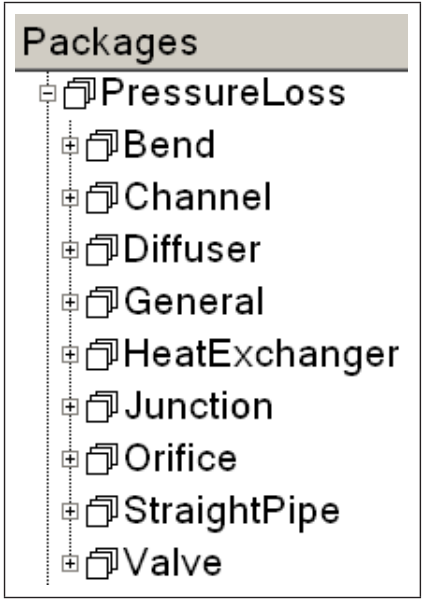

Figure 3: Package content of pressure loss devices in the FLuidDissipation library.

\section{Implementation of library}

\subsection{Functional approach}

The intention of the FLuidDissipation library is to create a base library for convective heat transfer and pressure loss calculations as dissipation effects of fluid flow. To ensure its interoperability with other thermohydraulic libraries (e.g. ModeliCA_FuUID) the following main aspects have been realised during modelling:

- Independence of thermo-hydraulic framework (applicable with other libraries)

- Literally use of function calls

- Input and/or output arguments of function calls are delivered by records (e.g. fluid properties, geometry parameters) 
Besides its interoperability this functional approach considers the ease of use at a maximum of numerical efficiency during system simulation. For example each function call only needs its target variables (e.g. mass flow rate, heat transfer coefficient or pressure loss) and one record with all other variables as input arguments. The intention for the future is to separate the dynamic variables of the input record from the constant ones (e.g. fluid properties from geometry parameters) due to numerical reasons (see 3.2). In addition functions using output records to monitor extra information like a failure status shall not be used for the integration into fluid models. These functions are best used for functions calls only.

The principles of how to implement convective heat transfer or pressure loss functions from FLUIDDISSIPATION into other thermo-hydraulic libraries can be done according to the following steps (here for pressure loss as example).

1. Use/Create model with missing pressure loss calculation (e.g. fluid interfaces for flow model)

2. Choose pressure loss function of interest

3. Choose corresponding pressure loss records

4. Build function-record construction

5. Assign record variables

This simplified implementation principle is shown in Fig.4.

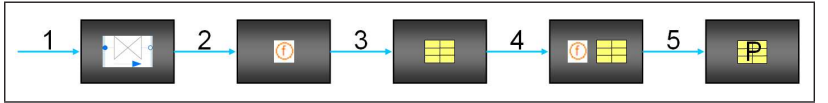

Figure 4: Principle implementation method to integrate FLUIDDISSIPATION functions into a thermohydraulic framework.

Integration examples of dissipation functions into MODELICA_FLUID as thermo-hydraulic framework are explained in detail in Sec.3.3. A description can also be found in [1] or in the GETTINGSTARTED section of the USERS GUIDE in the FLUIDDISSIPATION library itself.

\subsection{Numerical optimisation}

All convective heat transfer and pressure loss functions of the library are numerically optimised to provide efficient transient simulations. Measurement data for dissipation correlations are adapted into continuous functions. A smoothing function (see [3]) is used to ensure continuity and differentiability. For example the pressure loss of an overall fluid flow in a straight pipe is calculated via the correlations of the laminar and the turbulent regime with the smoothing function in between representing the transition regime.

An additional aspect to improve computational efficiency during system simulation can be to avoid numerical Jacobians. Numerical Jacobians are created in the translation process of a MoDELICA simulation tool out of an underlying equation system of a simulation model, if the tool is not able to create analytical Jacobians.

For instance the pressure loss in a flow model can be modelled as a compressible case ${ }^{1}$ or an incompressible case $^{2}$ to avoid additional nonlinear equations. An analytical or numerical Jacobians is then needed, if a compressible flow model has got a known mass flow rate at its interfaces instead of a pressure loss. Especially in large system simulations, the avoidance of nonlinear equations improves the performance of a simulation system because then there is no need for a nonlinear solver.

For pressure loss the FLUIDDISSIPATION library delivers both a compressible as well as an incompressible calculation for inverse calculations w.r.t. mathematical feasibility of its invertability. In an application flow model no additional nonlinear equations are created due to these functions if used correctly. Even if the pressure loss functions are used in a numerically unintended way by the user (e.g. the mass flow rate for a flow model is unknown in an incompressible case), all functions are able to provide analytical Jacobians to avoid the creation of numerical Jacobians.

\subsection{Integration of library into thermo- hydraulic framework using MODEL- ICA_FLUID library}

Convective heat transfer models and flow models have been created using FLUIDDISSIPATION functions and the ModeliCA_FLuID library as one example for the implementation into a thermo-hydraulic framework. These models calculate the convective heat transfer coefficient on the one hand and the mass flow rate or pressure loss on the other hand only. These base models have to be enhanced if additional features like balance equations or discretisation are needed.

The actual content of MoDELICA_FLuID base

\footnotetext{
${ }^{1}$ To calculate the mass flow rate out of a given pressure loss

${ }^{2}$ To calculate a pressure loss out of a given mass flow rate
} 
models provided by FLUIDDISSIPATION is shown in Fig.5. All dissipation correlations for convective heat

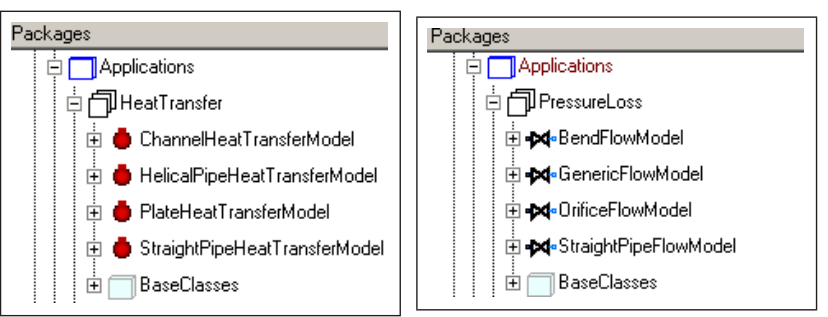

Figure 5: Application models using FLUIDDISSIPATION correlations and MODELICA_FLUID as thermo-hydraulic framework. Convective heat transfer models are shown in the left, whereas flow models are shown in the right.

transfer and pressure loss are grouped into its corresponding geometry device. For example if using a BENDFLOWMODEL the user can easily change a pressure loss calculation from an edged to a curved geometry. All dissipation correlations for each geometry device can be easily exchanged by choosing an intended application model via a drop-down menu in a parameter window ${ }^{3}$. After selection a corresponding record can be edited (e.g. for setting geometry parameters).

\subsubsection{Heat transfer}

An implementation of convective heat transfer functions into usable MODELICA_FLUID models is shown in Fig.6. Generally heat transfer correlations are modelled through replaceable heat transfer models. For example a PlateHeatTransferModel can be chosen to the laminar, turbulent or overall fluid flow regime. All replaceable heat transfer calculations of one energy device are stored separately its BASEClasses section.

Referring to Fig. 6 the calculation of the thermodynamic state (needed for the fluid properties) has to be calculated outside of the provided heat transfer models. Here the thermodynamic state can be assigned through an inner model provided at the same hierarchy as the used instance of a heat transfer model. The convective heat transfer coefficient $k c$ is calculated via a function call using its corresponding record with needed input variables like geometry parameters. The resulting heat flow rate $\dot{Q}_{\text {flow }}$ is calculated out of $k c$, heat transfer area $A_{k c}$ and temperature difference between thermal port and thermodynamic state.

\footnotetext{
${ }^{3}$ Here using Dymola from Dynasim as IDE
}

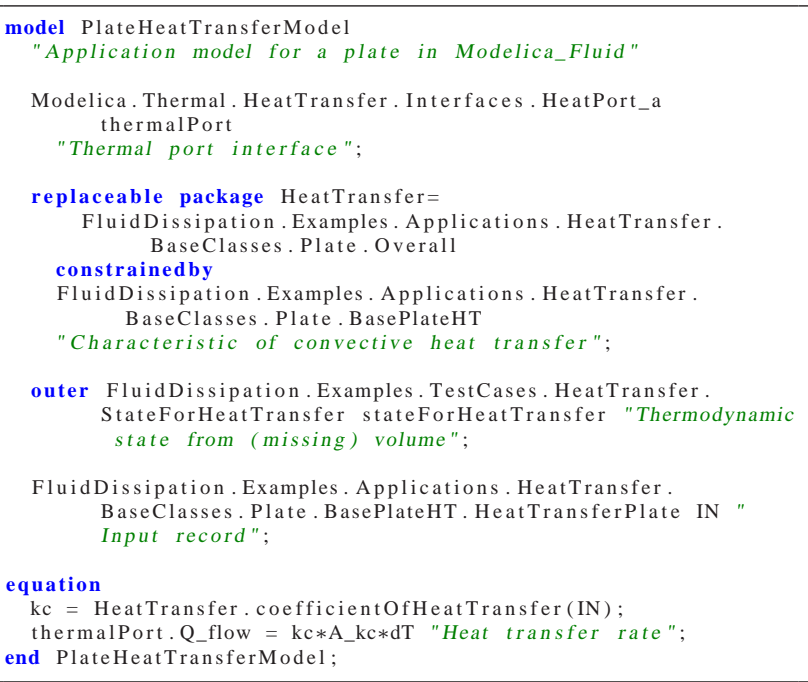

Figure 6: Relevant source code for a convective heat transfer model using FLUIDDISSIPATION correlations.

\subsubsection{Pressure loss}

An implementation of pressure loss functions using ModeliCA_Fluid is shown in Fig.7. Pressure loss correlations are modelled through replaceable pressure loss models. The modelling referring to Fig.7 allows

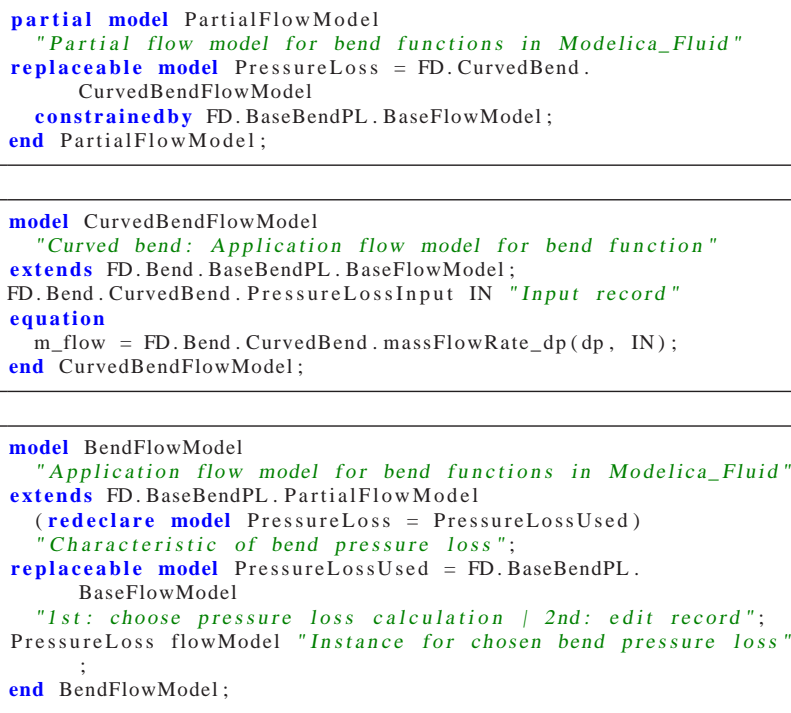

Figure 7: Relevant source code for a flow model using FLUIDDISSIPATION correlations implemented in ModELICA_FLUID as thermo-hydraulic framework.

to replace a flow model instance of each energy device by its alternatives stored in the BASECLASSES.

The calculation of pressure loss in a flow model can be implemented in dependence of targets. Then a com- 
pressible case or an incompressible case can be chosen to adjust an optimal numerical behaviour of a simulation model. Thus an inverse calculation without generating analytical Jacobians can be created automatically if supported by the MODELICA simulation tool.

\section{Verification and validation of li- brary}

All functions in the library are verified against its based literature or validated against other literature if available. These verifications or validations are documented in the online documentation of the library. The validation of convective heat transfer and pressure loss are exemplarily described for one phase and two phase flow afterwards.

For heat transfer all functions in the library delivers a local or mean convective heat transfer coefficient.

$$
k c=\frac{N u \cdot \lambda}{\text { Length }}
$$

$k c \quad$ as convective heat transfer coefficient $\left[\mathrm{W} / \mathrm{m}^{2} \cdot \mathrm{K}\right]$,

$\lambda \quad$ as heat conductivity of fluid $[\mathrm{W} / \mathrm{m} \cdot \mathrm{K}]$,

Length as characteristic length [m],

$\mathrm{Nu} \quad$ as Nusselt number [-].

The Nusselt number $N u$ results from the actual flow regime, fluid properties and geometry parameters.

The compressible or incompressible pressure loss calculation uses a (total) pressure loss coefficient according to Eq.2.

$$
\zeta_{t o t}=\frac{2 \cdot \Delta p_{t o t}}{\rho_{r e f} \cdot v_{r e f}^{2}}
$$

$\zeta_{\text {tot }} \quad$ as pressure loss coefficient $[\mathrm{W} / \mathrm{m} \cdot \mathrm{K}]$,

$\Delta p_{\text {tot }}$ as total pressure loss [Pa],

$\rho_{\text {ref }}$ as reference density $\left[\mathrm{kg} / \mathrm{m}^{3}\right]$,

$v_{\text {ref }}$ as reference velocity $[\mathrm{m} / \mathrm{s}]$.

The pressure loss coefficient $\zeta_{t o t}$ is defined as the ratio of the total pressure loss between the inlet and outlet of a device to the dynamic pressure in a reference section. The total pressure loss for overcoming the forces of hydraulic resistance is dissipated. Thus the state of flow undergoes a change. At adiabatic conditions the mechanical work of a fluid flow is converted into heat due to resistance forces ${ }^{4}$. However the temperature of the fluid does not change at a constant velocity then. The reason is that the work of expansion due to pressure loss is entirely converted into the

\footnotetext{
${ }^{4}$ Total energy as sum of thermal and mechanical energy remains constant.
}

work of overcoming the resistance forces. The heat generated by this mechanical work is compensated by cooling induced through this expansion.

The total pressure loss is arbitrary subdivided into local and frictional pressure losses even though they are physically inseparable.

$$
\Delta p_{t o t}=\Delta p_{l o c}+\Delta p_{f r i}
$$

$\Delta p_{t o t} \quad$ as total pressure loss [Pa],

$\Delta p_{\text {loc }}$ as local pressure loss [Pa],

$\Delta p_{f r i} \quad$ as frictional pressure loss [Pa].

The total pressure loss can only be assumed to be the difference in static pressures, if there is no change in the cross sectional area of an energy devices, no mixing or splitting occurs and geodetic pressure loss can be neglected. Otherwise total pressures shall be used for modelling because the pressure loss coefficient can also have negative values. For example a negative pressure loss coefficient can occur at the mixing of two fluid flows in a junction having different velocities. In this case the dynamic pressure difference of the fluid flow with the lower velocity is increased between the section before and after mixing.

\subsection{Heat transfer in an even gap}

The validation of convective heat transfer for an even gap is shown in Fig.8. The convective heat transfer co-

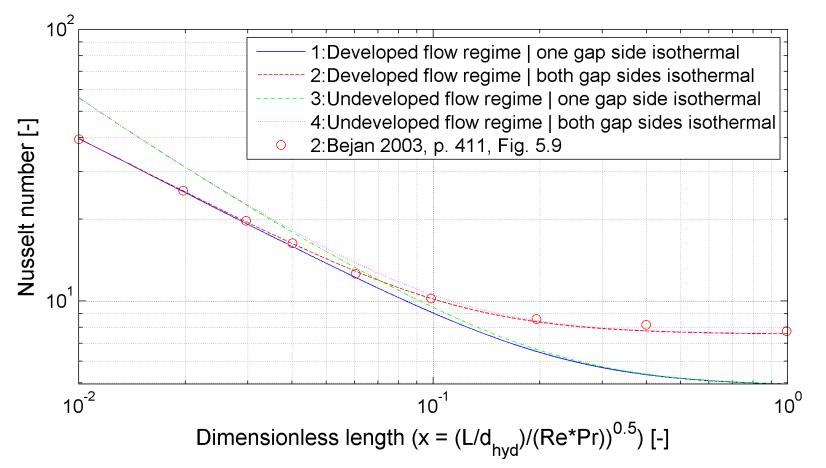

Figure 8: Validation for convective heat transfer of one phase laminar fluid flow in an even gap. The Nusselt number is shown in dependence of a dimensionless length at different boundary conditions. Simulation results are validated against Bejan [4].

efficient has been calculated for developed and undeveloped fluid flow with one or two sides of the gap being isothermal. The validation is shown for the mean Nusselt number in an even gap with isothermal walls at a developed fluid flow. There is a good correlation w.r.t. literature for this boundary condition. 


\subsection{Pressure loss in a curved bend}

The validation of pressure loss calculations is exemplarily shown for a curved bend in Fig.9.

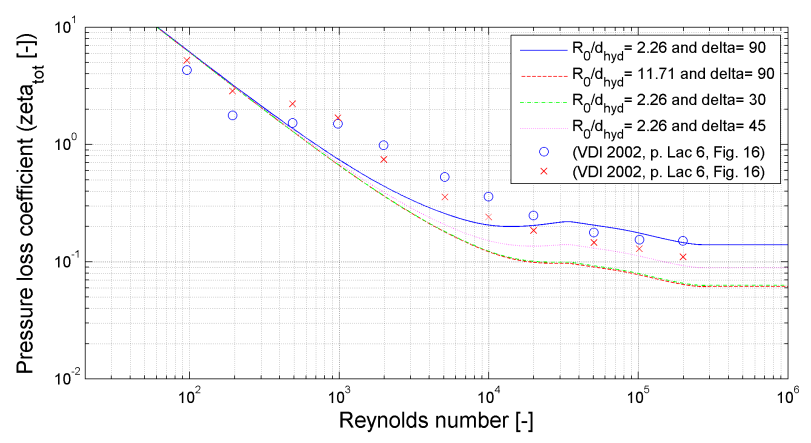

Figure 9: Validation of one phase overall fluid flow in a curved bend. The pressure loss coefficient is shown in dependence of the Reynolds number with relative curvature radii and angle of curvature of turning as parameter. Simulation results are validated against VDI [5].

The validation in Fig. 9 comparing the pressure loss correlations out of Idelchik [6] with data from VDI [5] shows the right physical behaviour of fluid flow through a curved bend. The results give a good prediction of pressure loss in the laminar and fully developed turbulent regime. Deviations in the transition regime are in an adequate range of uncertainty.

\subsection{Two phase flow in a horizontal straight pipe}

The validation of two phase heat transfer and pressure loss calculations is exemplarily shown for a horizontal straight pipe. The various flow regimes during boiling and condensation during two phase flow are shown in Fig.10. For example at stratified flow a fluid is divided into a separated vapour and liquid phase due to gravity. The actual two phase flow regime during boiling and condensation can be determined with known vapour fraction and mass flux out of a flow pattern map (e.g. from Steiner in VDI [5]). For describing two phase flow usually a heterogeneous or homogeneous approach is used. These simplified approaches differ in the calculation of the cross sectional void fraction. The complete range of two phase flow regimes shown in Fig.10 can be modelled with these approaches.

In the homogeneous approach gas and liquid phase have the same velocity. The homogeneous void frac-

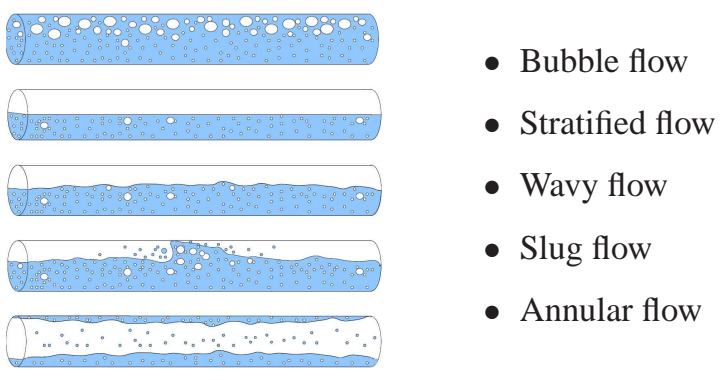

Figure 10: Flow regimes for two phase flow in horizontal straight pipes.

tion is calculated with Eq.4.

$$
\varepsilon_{\text {hom }}=\frac{1}{1+\left(\frac{1-\dot{x}}{\dot{x}}\right) \cdot \frac{\rho_{g}}{\rho_{l}}}
$$

$$
\begin{array}{ll}
\varepsilon_{h o m}=A_{g} / A_{g}+A_{l} & \text { as void fraction }[-], \\
\rho & \text { as density of a phase }\left[\mathrm{kg} / \mathrm{m}^{3}\right], \\
\dot{x}=\dot{m}_{g} / \dot{m}_{g}+\dot{m}_{l} & \text { as vapour fraction }[-], \\
A & \text { as cross sectional area of a phase }\left[\mathrm{m}^{2}\right], \\
\dot{m}_{g / l} & \text { as mass flow rate of a phase }[\mathrm{kg} / \mathrm{s}] .
\end{array}
$$

The fluid properties of the two phases are averaged using the homogeneous approach. This approach is best applicable for the bubble flow regime with gas bubbles uniformly dispersed in the liquid phase.

The heterogeneous approach describes the two phases separately. In this case each phase can flow with a constant but different mean velocity. The separated phases are described with the so called slip ratio $s$ as ratio of gaseous and liquid phase velocity. The heterogeneous void fraction is calculated from Eq.5.

$$
\varepsilon_{h e t}=\frac{1}{1+\left(\frac{1-\dot{x}}{\dot{x}}\right) \cdot \frac{\rho_{g}}{\rho_{l}} \cdot s}
$$

$$
\begin{array}{ll}
\varepsilon_{h e t} & \text { as void fraction }[-], \\
\rho & \text { as density of a phase }\left[\mathrm{kg} / \mathrm{m}^{3}\right], \\
s=v_{g} / v_{l} & \text { as slip ratio }[-], \\
v_{g / l} & \text { as velocity of a phase }[\mathrm{m} / \mathrm{s}]
\end{array}
$$

\subsubsection{Heat transfer at condensation}

The correlation of Shah [7] in Eq.6 is used to calculate the convective heat transfer during condensation in a horizontal straight pipe. In Shah [7] the heat transfer is assumed to take place only in the liquid phase. Therefore this correlation is best used for the annular flow regime, where the pipe wall is completely covered with liquid. The influence of the gaseous phase is 
described by a two phase multiplier.

$$
\alpha_{2 p h}=\alpha_{l} \cdot\left(1+\frac{3.8}{p_{r}^{0.38}} \cdot\left(\frac{\dot{x}}{1-\dot{x}}\right)^{0.76}\right)
$$

$$
\begin{array}{ll}
\alpha_{2 p h} & \text { as two phase heat transfer coefficient }\left[\mathrm{W} / \mathrm{m}^{2} \cdot \mathrm{K}\right], \\
\alpha_{l} & \text { as liquid heat transfer coefficient }\left[\mathrm{W} / \mathrm{m}^{2} \cdot \mathrm{K}\right], \\
p & \text { as actual pressure }[\mathrm{Pa}] \\
p_{c} & \text { as critical pressure }[\mathrm{Pa}], \\
p_{r}=p / p_{c} & \text { as reduced pressure }[-], \\
\dot{x} & \text { as vapour fraction }[-] .
\end{array}
$$

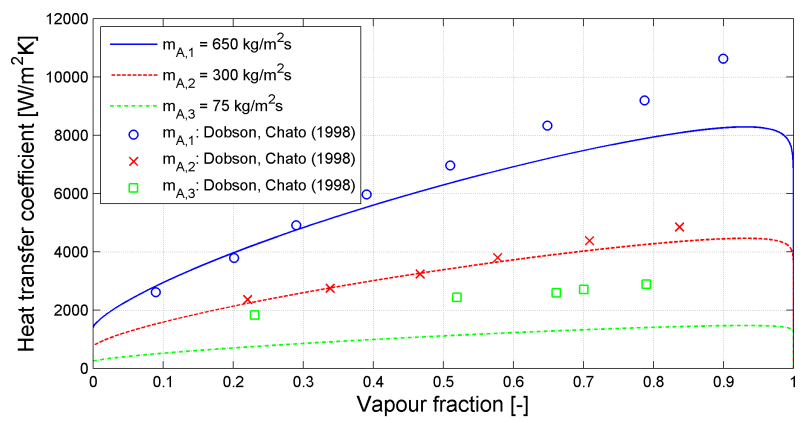

Figure 11: Validation of the local heat transfer coefficient during condensation in a horizontal straight pipe. The correlation of Shah [7] is validation against measurement results from Dobson/Chato [8] with the refrigerant R134a as medium.

In Fig.11 the Shah [7] correlation used for condensation in two phase flow has been validated against measurement results according to Dobson/Chato [8]. The refrigerant R134a has been used at a saturation temperature of $35^{\circ} \mathrm{C}$ and a mass flux between 75 to $650 \mathrm{~kg} / \mathrm{m}^{2} \cdot \mathrm{s}$. Generally these experimental data are underestimated by the correlation of Shah [7]. There are significant deviations for a small mass flux as well as for a very high mass flux and high vapour fraction. Deviations at a mass flux smaller than $130 \mathrm{~kg} / \mathrm{m}^{2} \cdot \mathrm{s}$ can be explained by having a different flow regime from the intended annular flow regime underlying the correlation of Shah [7]. For a moderate mass flux the validation shows a good prediction of the two phase heat transfer coefficient at annular flow regime.

\subsubsection{Heat transfer at boiling}

The correlation of Gungor/Winterton [9] is used to calculate the convective heat transfer during flow boiling in a horizontal straight pipe. This correlation expresses the physical effects of forced convection and nucleate boiling via a local two phase heat transfer coefficient (see Eq.7). The correlation can be used for subcooled and saturated flow boiling in horizontal and vertical straight pipes.

$$
\begin{aligned}
\alpha_{2 p h} & =E^{*} \cdot \alpha_{\text {conv }}+S^{*} \cdot \alpha_{\text {nucl }} \\
\alpha_{\text {conv }} & =\frac{0.023 \cdot\left[\operatorname{Re}_{l} \cdot(1-\dot{x})\right]^{0.8} \cdot \operatorname{Pr}_{l}^{0.4} \cdot \lambda_{l}}{d_{\text {hyd }}} \\
\alpha_{\text {nucl }} & =\frac{55 \cdot p_{r}^{0.12} \cdot q^{0.67}}{M^{0.5} \cdot\left(-\log _{10}\left(p_{r}\right)\right)^{0.55}}
\end{aligned}
$$

$\alpha_{2 p h} \quad$ as two phase heat transfer coefficient [ $\left.\mathrm{W} / \mathrm{m}^{2} \cdot \mathrm{K}\right]$,

$\alpha_{\text {conv }}$ as factor due to forced convection $\left[\mathrm{W} / \mathrm{m}^{2} \cdot \mathrm{K}\right]$,

$\alpha_{\text {nucl }}$ as factor due to nucleate boiling [W/ $\left./ \mathrm{m}^{2} \cdot \mathrm{K}\right]$,

$\lambda_{l}$ as liquid heat conductivity [W/m-K],

$d_{\text {hyd }}$ as diameter [m],

$E^{*} \quad$ as convection enhancement factor [-],

$S^{*} \quad$ as boiling suppression factor [-],

$M \quad$ as molar mass of medium $[\mathrm{kg} / \mathrm{mol}]$,

$p_{r} \quad$ as reduced pressure [-],

$\operatorname{Pr}_{l} \quad$ as liquid Prandtl number [-],

$\operatorname{Re}_{l} \quad$ as liquid Reynolds number [-],

$q \quad$ as heat flux $\left[\mathrm{W} / \mathrm{m}^{2}\right]$,

$\dot{x} \quad$ as vapour fraction $[-]$.

In Fig.12 the Gungor/Winterton [9] correlation used for flow boiling in two phase flow is validated against measurement results according to Kattan/Thome [10]. The refrigerant R134a has been used at a saturation temperature of $4.4^{\circ} \mathrm{C}$ and a mass flux between 100 to $299 \mathrm{~kg} / \mathrm{m}^{2} \cdot s$. The deviations in validation can be explained by considering the broad range of application for the Gungor/Winterton [9] correlation. Nevertheless there is an appropriate prediction of the heat transfer during flow boiling (e.g. for wavy to annular flow).

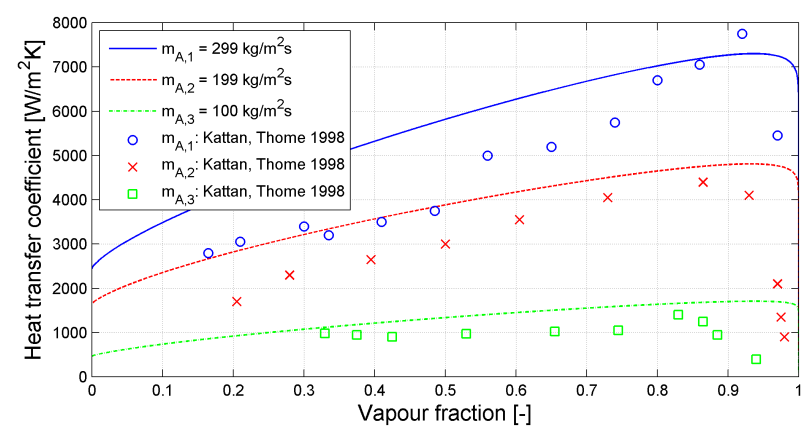

Figure 12: Validation of the local heat transfer coefficient at flow boiling in a horizontal straight pipe. The correlation of Gungor/Winterton [9] is validated against Kattan/Thome [10] with R134a as medium.

\subsection{Pressure loss}

The pressure loss in two phase flow for a horizontal straight pipe is calculated out of a momentum pressure 
loss and a frictional pressure loss.

$$
\Delta p_{2 p h}=\underbrace{\Delta p_{\text {mom }}}_{\text {momentum }}+\underbrace{\Delta p_{f r i}}_{\text {friction }}
$$

The momentum pressure loss due to a change of momentum of the gaseous and liquid phase is calculated according to Eq.11.

$$
\Delta p_{\text {mom }}=\left[\dot{m}_{A}^{2} \cdot\left(\frac{(1-\dot{x})^{2}}{\rho_{l} \cdot(1-\varepsilon)}+\frac{\dot{x}^{2}}{\rho_{g} \cdot \varepsilon}\right)\right]_{\dot{x}_{\text {inlet }}}^{\dot{x}_{\text {outlet }}}
$$

$\Delta p_{\text {mom }} \quad$ as momentum pressure loss $[\mathrm{Pa}]$,

$\varepsilon \quad$ as void fraction [-],

$\rho$ as density of a phase $\left[\mathrm{kg} / \mathrm{m}^{3}\right]$,

$\dot{m}_{A} \quad$ as total mass flux $\left[\mathrm{kg} / \mathrm{m}^{2} \cdot \mathrm{s}\right]$,

$\dot{x} \quad$ as vapour fraction [-].

The momentum pressure loss is calculated by the difference of using Eq.11 with a vapour fraction at the outlet and the inlet of the horizontal straight pipe. The void fraction can be described either by the homogeneous or heterogeneous approach (see Sec.4.3).

The frictional two phase pressure loss of a horizontal straight pipe is determined by the correlation of Friedel [11]. This correlation uses the heterogeneous approach for all two phase flow regimes. The two phase pressure loss $\Delta p_{\text {fri }}$ results from the frictional pressure loss of the liquid phase $\Delta p_{f r i, l}$ and a two phase multiplier $R$. For the calculation of the liquid frictional pressure loss all the mixture is assumed to flow as liquid.

$$
\Delta p_{f r i}=\Delta p_{f r i, l} \cdot R
$$

The two phase multiplier according to Friedel [11] is calculated in Eq.13.

$$
\begin{aligned}
R= & (1-\dot{x})^{2}+\dot{x}^{2} \cdot \frac{\zeta_{g}}{\zeta_{l}} \cdot \frac{\rho_{l}}{\rho_{g}}+3.43 \cdot \dot{x}^{0.69} \\
\cdot & (1-\dot{x})^{0.24} \cdot\left(\frac{\rho_{l}}{\rho_{g}}\right)^{0.8} \cdot\left(\frac{\eta_{g}}{\eta_{l}}\right)^{0.22} \\
& \left(1-\frac{\eta_{g}}{\eta_{l}}\right)^{0.89} \cdot \mathrm{Fr}_{l}^{-0.047} \cdot W e_{l}^{-0.033}
\end{aligned}
$$

$\eta_{g / l} \quad$ as dynamic viscosity of a phase $[\mathrm{kg} / \mathrm{m} \cdot \mathrm{s}]$,

$\rho_{g / l} \quad$ as density of a phase $\left[\mathrm{kg} / \mathrm{m}^{3}\right]$,

$\zeta_{g / l} \quad$ as Darcy friction factor of a phase [-],

$F r_{l} \quad$ as liquid Froude number [-],

$R \quad$ as two phase multiplier [-],

$R e_{g / l} \quad$ as Reynolds number of a phase [-],

$W e_{l}$ as liquid Weber number [-],

$\dot{x} \quad$ as vapour fraction $[-]$.
The Darcy friction factor $\zeta_{g / l}$ for each phase is calculated in dependence of the corresponding Reynolds number in Eq. 14.

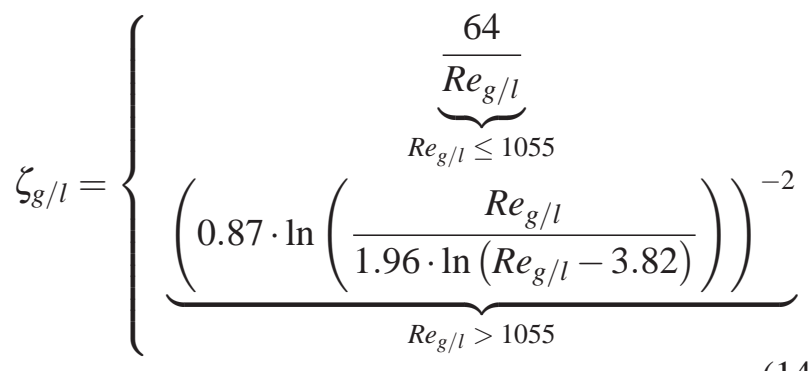

In Fig.13 the Friedel [11] correlation used for two phase pressure loss is validated against measurement results according to SINTEF [12]. As medium $\mathrm{CO}_{2}$ at a saturation temperature of $10^{\circ} \mathrm{C}$ and a mass flux between 200 to $400 \mathrm{~kg} / \mathrm{m}^{2}$.s has been used. Both momentum and frictional pressure loss at mean fluid properties are considered.

In a technical report of SINTEF [12] several different pressure loss correlations are validated against experimental pressure loss data for evaporation of $\mathrm{CO}_{2}$. The correlation of Friedel [11] delivers the most accurate results with a mean deviation of $22 \%$. The validation of the implemented two phase pressure loss shows good agreement according to SINTEF [12]. Nevertheless the pressure loss is underestimated at a low vapour fraction.

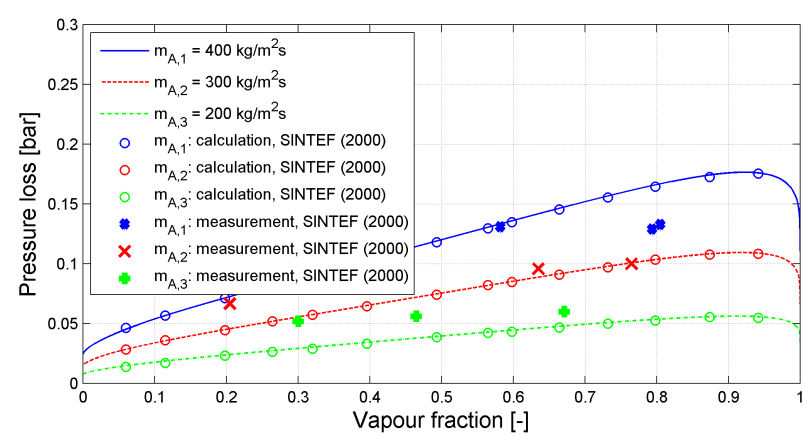

Figure 13: Validation of local pressure loss during flow boiling in a horizontal straight pipe. The correlation of Friedel [11] is validated against measurement results from SINTEF [12] with $\mathrm{CO}_{2}$ as medium.

\section{Industrial applications}

This section gives examples for the usage of heat transfer and pressure loss correlations from FLUIDDISSIPATION in industrial system simulation. The dissipation correlations of the library are implemented into 
different thermo-hydraulic frameworks like MoDELICA_FLUID.

\subsection{Environmental control system}

The evaluation of the dissipation correlations from FLUIDDISSIPATION is also carried out by Dassault Aviation as partner in the EuroSysLib project. In Fig.14 measurement data from a physical test bench representing an evaluation version of an aircraft have been compared to a corresponding simulation model using FLUIDDissipation. There is a good agreement for first evaluation results of subsystems from the environmental control system in Fig.14.

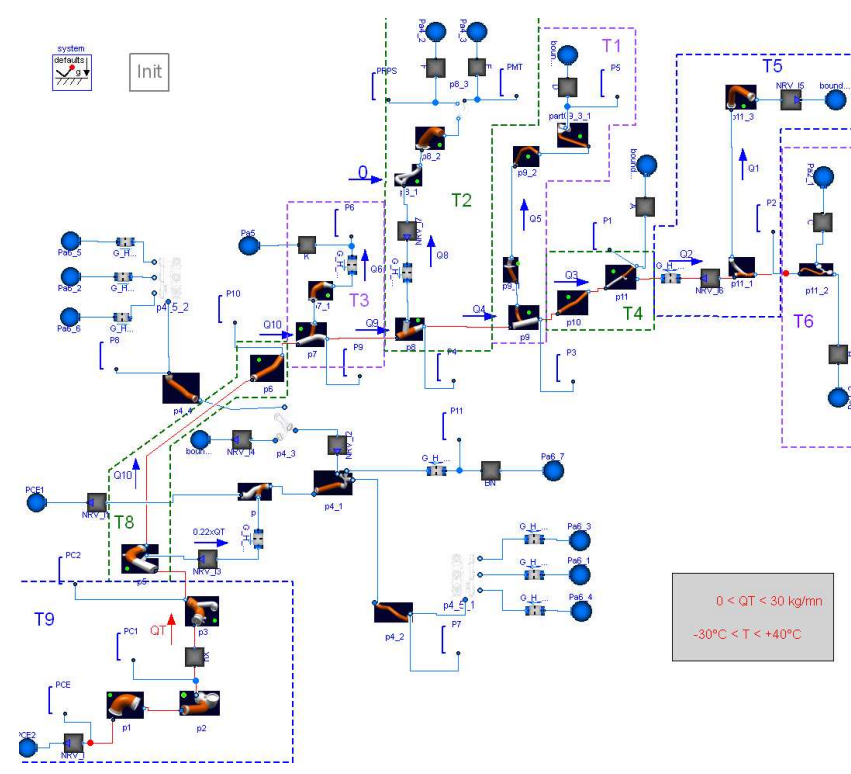

Figure 14: Evaluation of an environmental control system using FLUIDDISSIPATION carried out by Dassault Aviation.

\subsection{Supplemental cooling system}

A simulation model of an indirect cooling cycle for an air distribution circuit used for electrical equipment cooling is shown in Fig.15. Here dissipation correlations are integrated into a thermo-hydraulic library called HYDRONICS. Liquid water is used as cooling medium. The aim of this system is to cool different air flows in two heat exchanger. The optimal distribution of cold water is achieved by two control valves.

\subsection{Aircraft engine feeding system}

A simulation model of an aircraft engine feeding system is shown in Fig.16. Here FluidDissipation

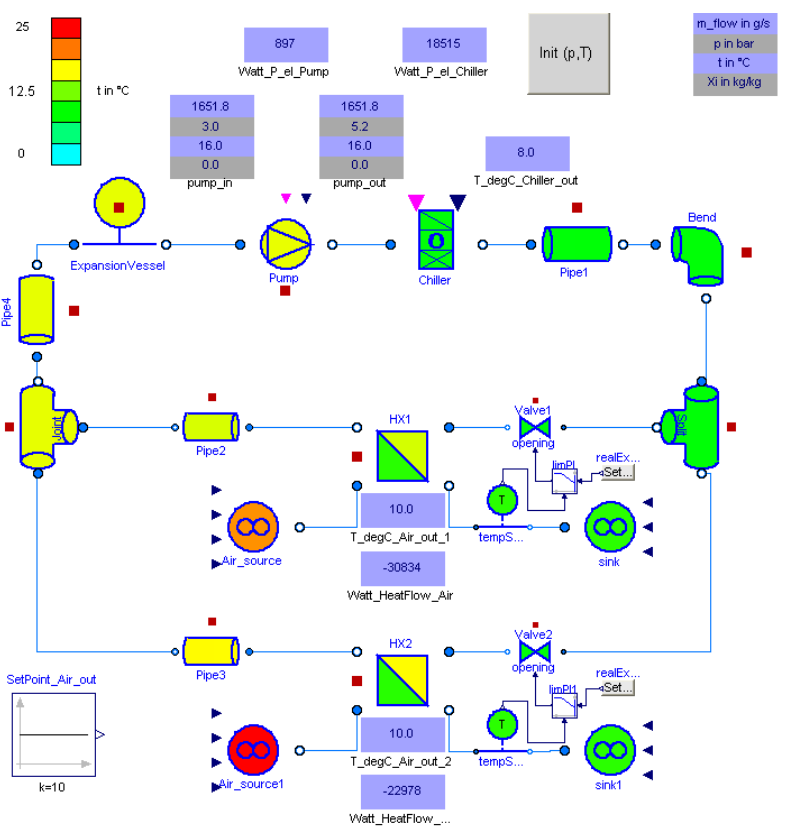

Figure 15: Simulation model of indirect cooling cycle for an air distribution circuit used for electrical equipment cooling. FLUIDDISSIPATION correlations are implemented in HYDRONICS as library.

is implemented into ModELICA_FLUID as thermohydraulic framework. An incompressible medium is used for the engine fuel (Jet A-1). The aim of this system is to control distribution of fuel for aircraft engines out of pressurised tanks. The simulation model analyses the system behaviour after the opening of the intercommunication valve in the emergency case. In the nominal case an intercommunication valve and the dump valve is closed. In this case the left hand pump feeds the left engine as the right hand pump does for the right engine. Both intercommunication valve and dump valve are opened if fuel has to be dumped from the aircraft in an emergency case.

\section{Summary}

The FLUIDDISSIPATION library offers a broad range of verified and validated convective heat transfer and pressure loss correlations for energy system. The library has been numerically optimised for efficient transient simulations. All correlations are documentation in detail describing its scope of usage.

ModELICA_FLUID models are provided as examples for the application into a thermo-hydraulic framework.

Scope, implementation concept, numerical challenges, verification and validation of the FLUIDDISSI- 


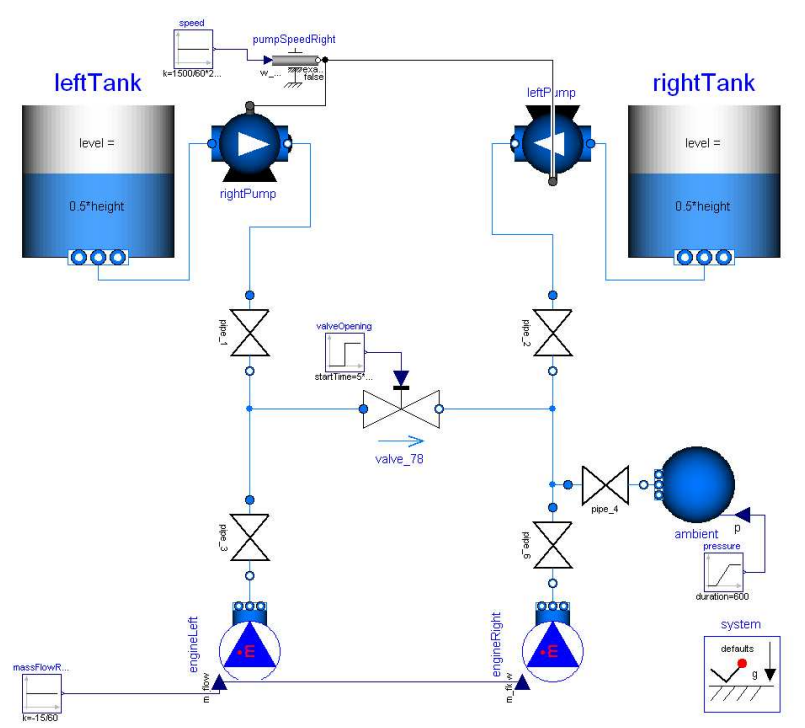

Figure 16: Simulation model of an aircraft engine feeding system using flow models with FLUIDDISSIPATION correlations and MODELICA_FLUID as thermo-hydraulic framework.

PATION library has been described as well as industrial applications of thermo-hydraulic energy systems.

FLUIDDISSIPATION is free for commercial and non-commercial applications and it can be used under the terms of the Modelica Licence 2.

\section{Acknowledgement}

The authors like to thank Eric Thomas for his evaluation results and Nina Peci for her contribution of two phase flow correlations.

The FluidDissipation library is developed within the European research project EuroSysLib-D funded by German Federal Ministry of Education and Research (promotional reference 01IS07022B). The project is started in October 2007 and will end in March 2010. The authors bear the sole responsibility for the content of this publication.

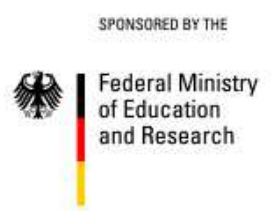

\section{References}

[1] T. Vahlenkamp and S. Wischhusen. Fluiddissipation - a centralised library for modelling of heat transfer and pressure loss. In B. Bachmann, edi- tor, Proceedings of the 6th International Modelica Conference, volume 1, pages 173-178, Bielefeld, Germany, March 2008.

[2] Casella, Francesco et al. The Modelica Fluid and Media library for modeling of incompressible and compressible thermo-fluid pipe networks. In Proceedings of the 5th International Modelica Conference, pages 631-640, Linköping, Sweden, 2006. The Modelica Association.

[3] S. Wischhusen. Dynamische Simulation zur wirtschaftlichen Bewertung von komplexen Energiesystemen. $\mathrm{PhD}$ thesis, Technische Universität Hamburg-Harburg, 2005.

[4] A Bejan and A.D. Kraus. Heat Transfer handbook. John Wiley \& Sons, 2nd edition, 2003.

[5] VDI. VDI - Wärmeatlas: Berechnungsblätter für den Wärmeübergang. Springer Verlag, 9th edition, 2002.

[6] I. E. Idelchik. Handbook of hydraulic resistance. Jaico Publishing House, Mumbai, 3rd edition, 2006.

[7] M.M. Shah. A general correlation for heat transfer during film condensation inside pipes. Int. J. Heat Mass Transfer, 22:547-556, 1979.

[8] M.K. Dobson and J.C. Chato. Condensation in smooth horizontal tubes. Journal of Heat Transfer, 120:193-213, 1998.

[9] K.E. Gungor and R.H.S. Winterton. A general correlation for flow boiling in tubes and annuli. Int. J. Heat Mass Transfer, 29:351-358, 1986.

[10] N. Kattan and J.R. Thome. Flow boiling in horizontal pipes: Part 2 - new heat transfer data for five refrigerants. Journal of Heat Transfer, 120:148-155, 1998.

[11] L. Friedel. Improved friction pressure drop correlations for horizontal and vertical two phase pipe flow. 3R International, 18:485-491, 1979.

[12] R. Pettersen, J.; Rieberer and S.T. Munkejord. Heat transfer and pressure drop characteristics of evaporating carbon dioxide in microchannel tubes. Technical report, SINTEF Energy Research, 2000.

[13] D.S. Miller. Internal flow systems, volume 5th of BHRA Fluid Engineering Series. BHRA Fluid Engineering, 1984. 